\title{
FGF-dependent regulation of VEGF receptor 2 expression in mice
}

\author{
Masahiro Murakami, ${ }^{1}$ Loc T. Nguyen, ${ }^{1}$ Kunihiko Hatanaka, ${ }^{1}$ William Schachterle, ${ }^{2}$ \\ Pei-Yu Chen, ${ }^{1}$ Zhen W. Zhuang, ${ }^{1}$ Brian L. Black, ${ }^{2}$ and Michael Simons ${ }^{1,3}$
}

\begin{abstract}
${ }^{1}$ Section of Cardiovascular Medicine, Department of Internal Medicine, Yale University School of Medicine, New Haven, Connecticut, USA 2UCSF, San Francisco, California, USA. ${ }^{3}$ Department of Cell Biology, Yale University School of Medicine, New Haven, Connecticut, USA.
\end{abstract}

\begin{abstract}
Numerous studies have suggested a link between the angiogenic FGF and VEGF signaling pathways; however, the nature of this link has not been established. To evaluate this relationship, we investigated VEGF signaling in ECs with disrupted FGF signaling in vitro and in vivo. ECs lacking FGF signaling became unresponsive to VEGF, caused by downregulation of VEGF receptor 2 (VEGFR2) expression after reduced Vegfr 2 enhancer activation. FGF mediated VEGFR2 expression via activation of Erk1/2. Transcriptional analysis revealed that Ets transcription factors controlled VEGFR2 expression in an FGF- and Erk1/2-dependent manner. Mice with defective FGF signaling exhibited loss of vascular integrity and reduced vascular morphogenesis. Thus, basal FGF stimulation of the endothelium is required for maintenance of VEGFR2 expression and the ability to respond to VEGF stimulation and accounts for the hierarchic control of vascular formation by FGFs and VEGF.
\end{abstract}

\section{Introduction}

New vessel formation is a multistep process requiring integrated actions of a number of angiogenic growth factors. Among them, the FGF and VEGF families are the most potent promoters of angiogenesis. Although synergistic action of FGF and VEGF has been observed and the crosstalk of the 2 families has been suggested as an essential regulatory step in vascular formation, the key details of this mechanism, which have long been a fundamental question in vascular biology, are not understood (1).

The FGF family is one of the largest and evolutionarily preserved growth factor families; FGFs are capable of acting on a variety of cell types. They are critical in early embryonic development and precede the appearance of VEGF signaling. In adults, FGFs play key roles in neovascularization, wound healing, and cancer (2-4). One of the characteristic features of FGF signaling is the context specificity of action, producing divergent biological effects depending on the effector cell type and the dose, duration, or timing of action (5). This complex biology of FGFs suggests that they play a regulatory role in many biological settings by influencing multiple cellular components (4).

Studies of the biological role of the FGF system have been complicated by the great redundancy among FGFs and by indispensable roles played by FGFR1 and FGFR2 in embryonic development (6). A noteworthy recent discovery has been the demonstration of an essential role played by endothelial FGF signaling in the maintenance of blood vessels (7). At the same time, VEGF and its receptors, VEGF receptor 1 (VEGFR1) and VEGFR2, play a key role in vascular development and maintenance of the adult vasculature.

These observations suggest a tight integration of FGF and VEGF signaling in the endothelium. To study this interaction, we used several complementary approaches to block FGF signaling in ECs in vitro and in vivo and observed the effect of this inhibition on VEGF signaling and VEGF-induced biological responses. Suppression of endothelial FGF signaling, either by depletion of exogenous

Conflict of interest: The authors have declared that no conflict of interest exists. Citation for this article: J Clin Invest. 2011;121(7):2668-2678. doi:10.1172/JCI44762.
FGFs or by shutdown of all FGF receptor signaling using a dominant-negative construct, resulted in impairment of VEGF signaling caused by pronounced reduction in VEGFR2 expression, which was in turn caused by decreased Vegfr 2 enhancer activity. In vivo, this was manifested by increased vascular permeability and impaired angiogenic and arteriogenic responses. The molecular defect was traced to reduced activation of Ets transcription factors induced by FGFdependent Erk1/2 activation and binding to the Vegfr 2 enhancer Ets site contained within the recently described FOX:ETS motif (8). Our data demonstrated the precise mechanism of the crosstalk between the 2 cardinal angiogenic growth factor families and how they coordinately regulate the neovascularization process.

\section{Results}

FGF signaling controls EC responsiveness to VEGF. To test the hypothesis that FGF signaling controls EC responsiveness to VEGF and thus regulates VEGF function, we used a cytoplasmic truncated form of FGF receptor 1 (FGFR1) able to heterodimerize with all FGFRs as a dominant-negative construct (FGFR1DN), thereby suppressing overall FGF signaling $(7,9,10)$. Bovine aortic ECs (BAECs) transduced with Ad vector encoding FGFR1DN (Ad-FGFR1DN) showed, as expected, decreased Erk1/2 and Akt phosphorylation in response to FGF stimulation; however, the response to VEGF-A was equally impaired in these cells (Figure 1A). Western blot analyses of VEGF receptor expression demonstrated a marked reduction in levels of VEGFR2, but not VEGFR1 (Figure 1A). Reduced VEGFR2 expression by FGF inhibition was also confirmed in Ad-FGFR1DN-transduced primary mouse aortic ECs (MAECs; Supplemental Figure 1A; supplemental material available online with this article; doi:10.1172/JCI44762DS1), which indicates that this regulation is not specific to BAECs.

To demonstrate the physiologic significance of this reduction in VEGFR2 levels, we studied EC NO production in response to VEGF. Expression of FGFR1DN markedly reduced VEGF-A-induced cGMP accumulation compared with that in control Ad-GFPinfected cells (Figure 1B), which implies that FGF signaling is functionally important to this process. 
A

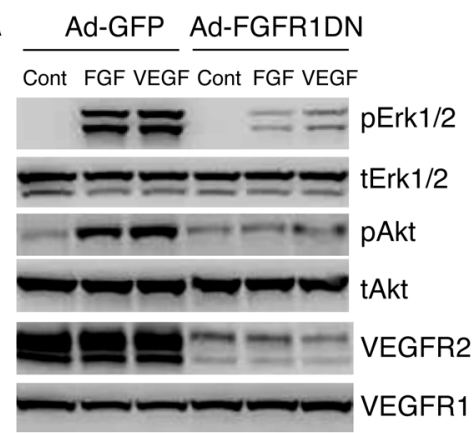

D

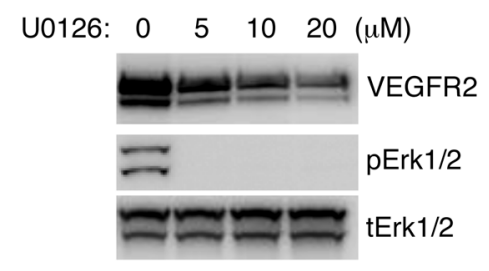

\section{G} Ad-Null Ad-ME-LA Ad-ME Ad-Null Ad-ME-LA Ad-ME

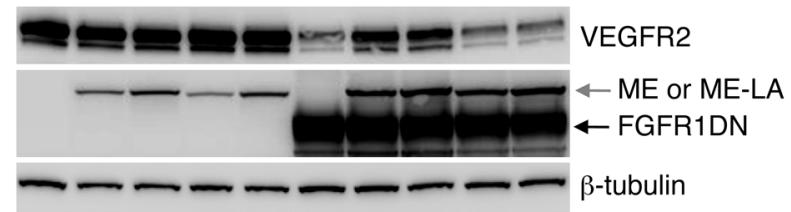

B

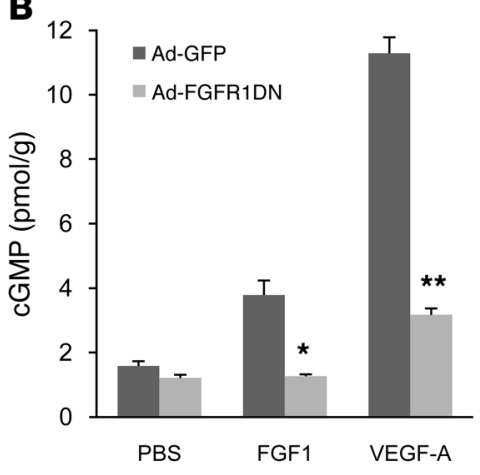

E

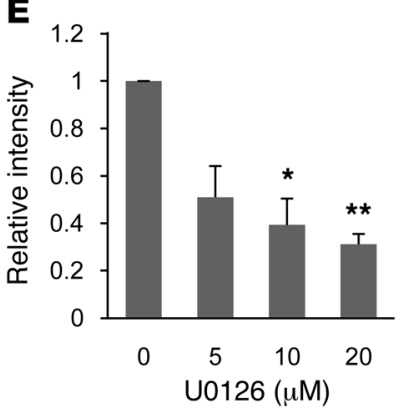

C

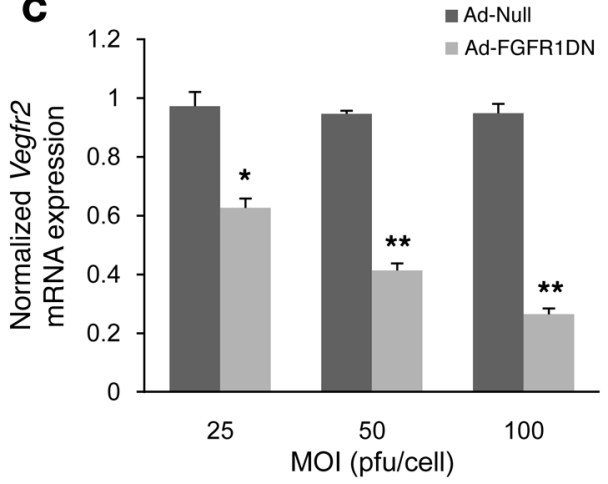

F

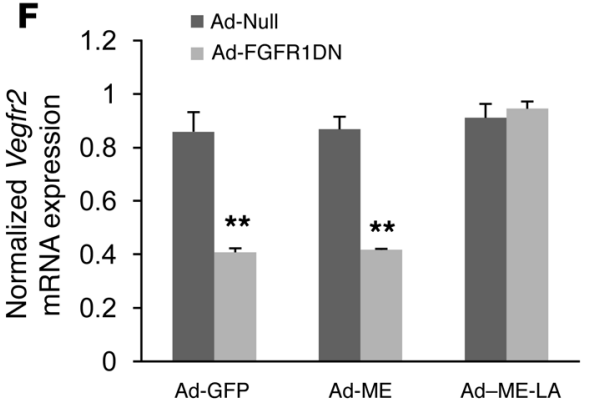

Figure 1

FGF regulation of VEGF signaling and VEGFR2 expression. (A) Western blot of total cell lysates of BAECs transduced with Ad-GFP or Ad-FGFR1DN and stimulated with FGF1 (50 ng/ml) or VEGF-A ( $80 \mathrm{ng} / \mathrm{ml})$ for 5 minutes. Cont, control; p-, phosphorylated; t-, total. (B) Quantitative analysis of cellular cGMP levels $(n=3)$. (C) Quantitative RT-PCR analysis of total RNA isolated from BAECs. Vegfr2 mRNA levels were measured with real-time PCR and normalized to Gapdh expression. Values denote abundance relative to that of untreated BAECs (assigned as 1). (D) Western blotting of total cell lysates isolated from BAECs treated with U0126 at different concentrations for 6 hours in normal growth medium. (E) Quantitative analysis of VEGFR2 levels. (F) BAECs were first transduced with Ad-Null or Ad-FGFR1DN, then the next day with Ad-ME or Ad-ME-LA. Quantitative PCR analysis of VEGFR2 expression was performed $(n=3)$. (G) Western blotting of total cell lysates showed increased VEGFR2 levels in Ad-FGFR1DN-transduced cells after Ad-ME-LA treatment. ME-LA and FGFR1DN (gray and black arrows, respectively) were detected with an $\mathrm{HA}$ antibody. ${ }^{*} P<0.05,{ }^{\star \star} P<0.01$ versus respective control.

To confirm that other means of FGF inhibition have a similar effect on VEGFR2 expression, we used soluble FGF receptors (sFGFRs), which are able to bind and trap FGF ligands in the extracellular space, and knockdown of FRS2, a key FGFR signaling regulator. We previously evaluated the efficacy of sFGFRs, and sFGFR1-IIIc, with its ability to bind many FGF ligands, is the most potent FGF inhibitor (7). Similar to Ad-FGFR1DN, Ad-sFGFR1-IIIc reduced Vegfr2 mRNA in BAECs in a dose-dependent manner (Supplemental Figure 1, B and C). Knockdown of FRS $2 \alpha$ in human umbilical artery ECs also resulted in decreased VEGFR2 expression (Supplemental Figure 1D).

We then tested whether FGF signaling is equally necessary for the maintenance of VEGFR2 expression in microvascular ECs. Transduction of mouse lung microvascular ECs with either AdFGFR1DN or Ad-sFGFR1-IIIc resulted in a decrease in VEGFR2 levels similar to that observed in BAECs or human umbilical artery ECs (Supplemental Figure 1, E-G).
We next tested whether FGF signaling controls VEGFR2 expression at the transcriptional or posttranscriptional level. Quantitative analyses of Vegfr 2 mRNA from Ad-FGFR1DN-transduced BAECs demonstrated a dose-dependent decrease in mRNA abundance, whereas the control virus had no effect (Figure 1C), suggestive of a primary transcription effect. At the same time, VEGFR2 protein half-life was not altered by suppression of FGF signaling (Supplemental Figure 2, A and B). Moreover, inhibition of proteasomal degradation using MG132 did not have any effect on VEGFR2 protein levels in these settings (Supplemental Figure 2C).

FGF-induced Erk1/2 activation mediates VEGFR2 expression. To further analyze the mechanism of FGF-mediated regulation of VEGFR2 expression, we investigated downstream signaling after FGFR activation. Upon binding to FGFR, FGF triggers activation of 3 major signaling pathways: Ras-MAPK, PI3K-Akt, and PLC $\gamma$-PKC (6). Whereas pharmacological inhibition of MEK1 and MEK2 by U0126 markedly 
A

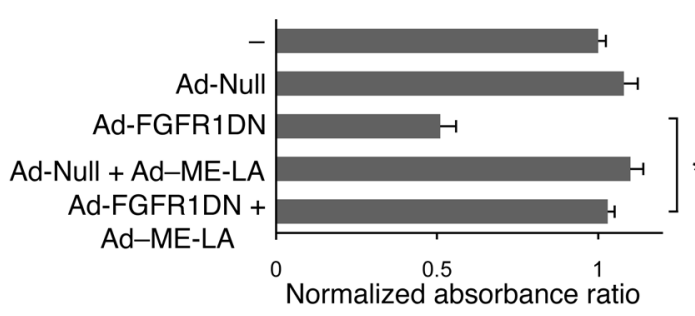

B

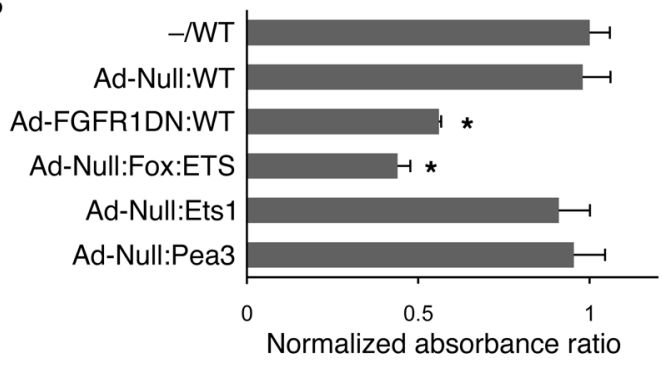

C

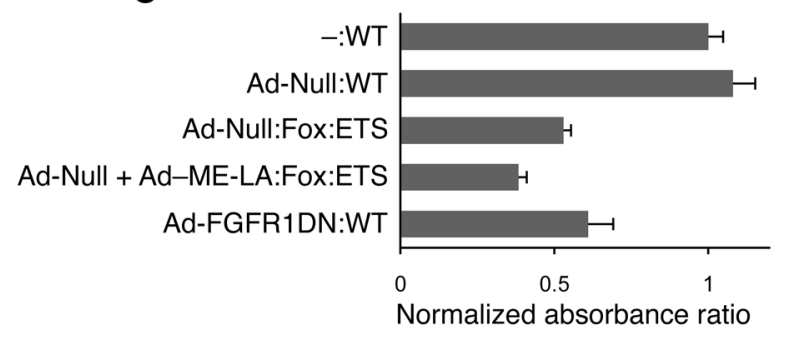

D

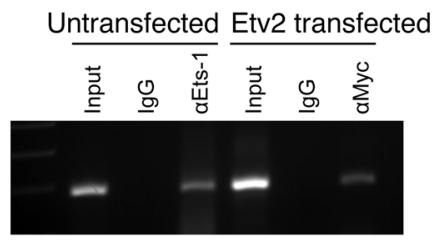

E

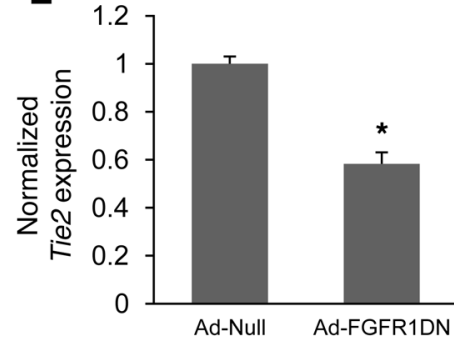

$\mathbf{F}$

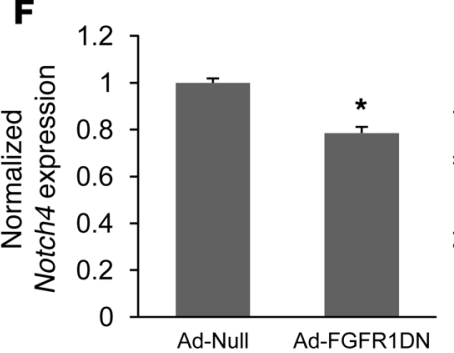

G

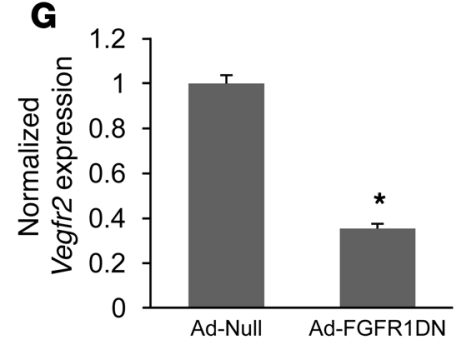

H

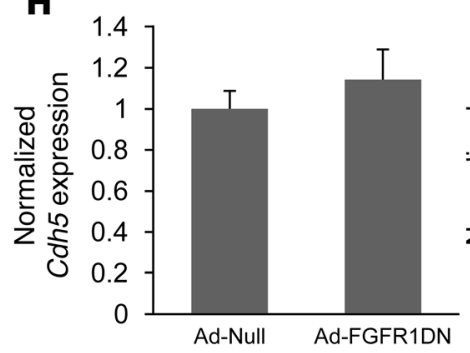

I

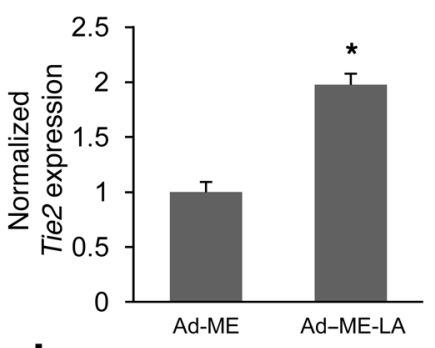

J

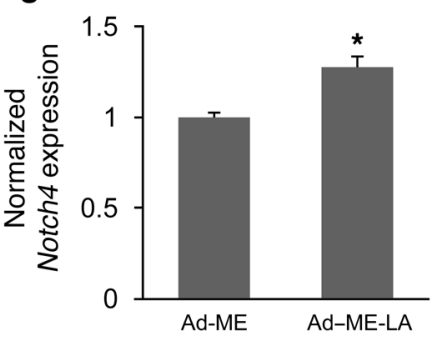

K

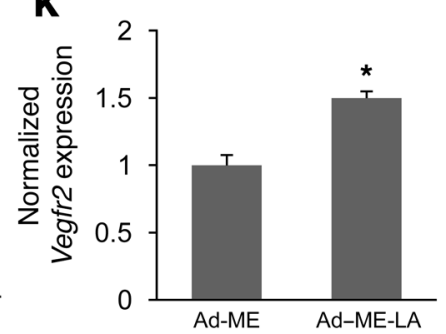

L

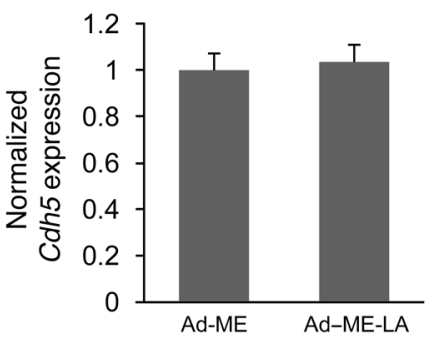

Figure 2

FGF signaling controls VEGFR2 enhancer activity and expression of other endothelial genes. (A) BAECs were first transduced with Ad-Null or Ad-FGFR1DN and subsequently with Ad-ME-LA, and transfected with the luciferase reporter construct containing Vegfr2 first intron enhancer and minimal promoter. (B) Luciferase reporter assay using BAECs transduced with Ad-Null or Ad-FGFR1DN and transfected with the WT Vegfr2 enhancer or minimal promoter constructs carrying Ets binding site mutations (Pea3, G56T_G57T; Ets1, G303T_G304T; FOX:ETS, C378A_ C379A). (C) Decreased enhancer activity by mutation of the FOX:ETS site was not rescued by constitutively active Erk2. (D) ChIP assay of Ets 1 and Etv2 binding to VEGFR2 enhancer in BAECs (untransfected or transfected with Myc-tagged Etv2). Input DNA, sample representing total input chromatin (1\%). (E-H) Quantitative RT-PCR analysis of total RNA isolated from BAECs transduced with Ad-Null or Ad-FGFR1DN. Values denote abundance relative to that of Ad-Null (assigned as 1). (I-L) Quantitative RT-PCR analysis of total RNA isolated from BAECs transduced with Ad-ME or Ad-ME-LA. ${ }^{*} P<0.05$ versus respective control or as indicated by brackets, Student's $t$ test.

attenuated VEGFR2 expression in a dose- and time-dependent manner (Figure 1, D and E, and Supplemental Figure 3, A and B), inhibition of PI3K by LY294002 had no effect (Supplemental Figure 3C). To study the involvement of Erk1/2 in regulation of VEGFR2 expression, we used Ad vectors encoding Erk2 fused to the low-activity form of its upstream regulator, Mek1. Whereas the Mek1-Erk2 (ME) hybrid protein is retained in the cytoplasm because of the presence of a nuclear export sequence, mutation of the 4 leucines in the export sequence to alanines (Mek1-Erk2-LA [ME-LA]) dramatically increases its catalytic activity with concomitant nuclear translocation $(11,12)$. Transduction of Ad-ME-LA, but not Ad-GFP or Ad-ME, into FGFR1DN-expressing BAECs restored Vegf 2 mRNA and protein expression to control levels (Figure 1, F and G), which indicates that regulation of VEGFR2 expression by FGF is mediated through an Erk1/2-dependent signaling mechanism.

Transcriptional regulation of FGF-induced VEGFR2 expression. Transcriptional control of VEGFR2 expression is complex and realized by multiple mechanisms $(13,14)$. Since Erk $1 / 2$ is known to activate several transcription factors by increasing their phosphorylation (15), we measured transcriptional activity of a reporter construct 
A
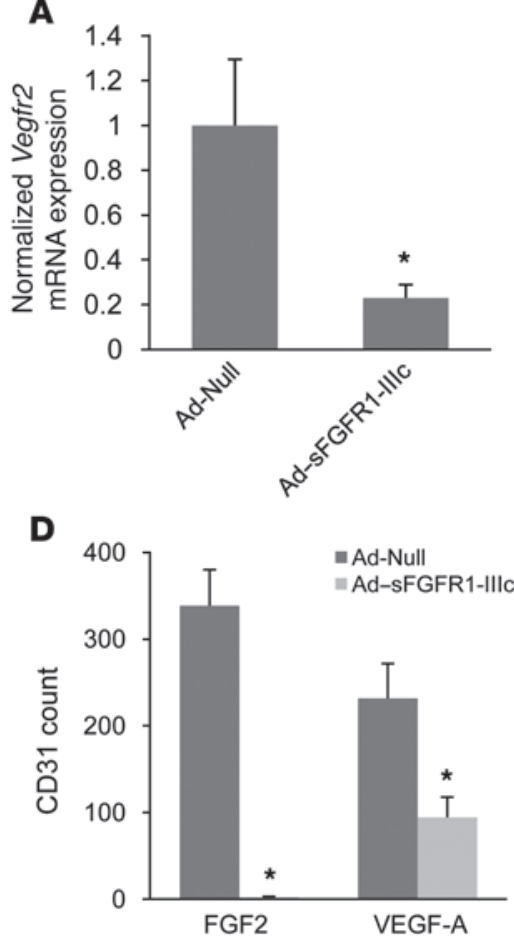

$\mathbf{F}$

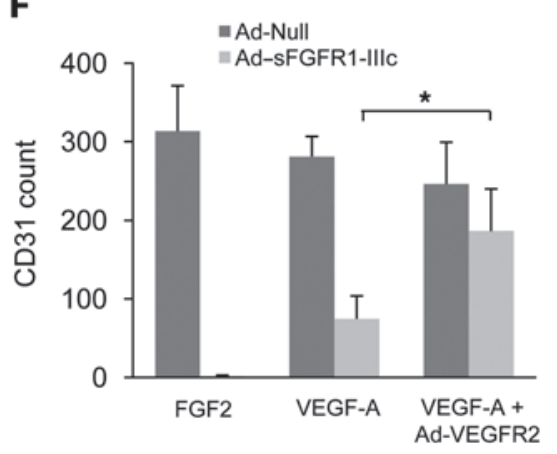

B

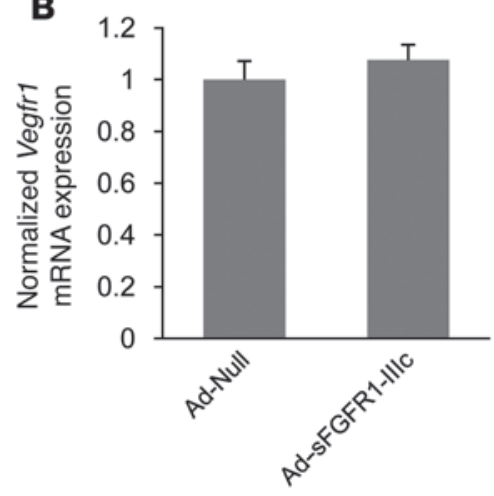

C

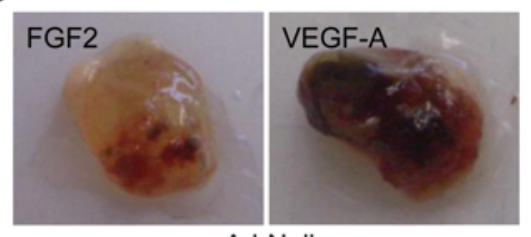

Ad-Null

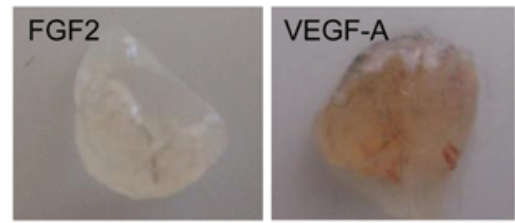

Ad-sFGFR1-IIIc
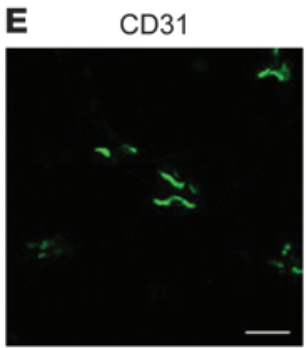

Flag (VEGFR2)
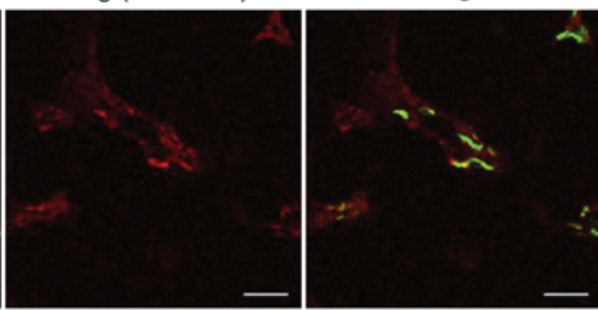

G

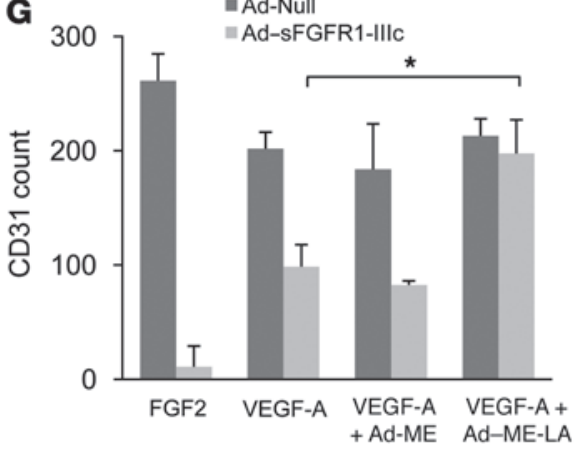

Figure 3

FGF signaling controls VEGF-induced angiogenesis. (A and B) Quantitative real-time PCR analysis of Vegfr2 (A) and Vegfr1 (B) mRNA expression in adductor muscle. Total RNA was isolated from muscle tissues of C57BL/6 mice injected with Ad-Null or Ad-sFGFR1-IIIc $\left(5 \times 10^{10}\right.$ viral particles) harvested 7 days after Ad injection ( $n=3$ per group). (C) Matrigel plugs, mixed with either FGF2 or VEGF-A, were placed subcutaneously in C57BL/6 mice injected with Ad-Null or Ad-sFGFR1-IIlc 7 days before plug implantation. (D) Quantitative analysis of Matrigel plug assay. Sections of Matrigel plugs were stained for CD31, and the number of vessels was counted ( $n=3$ per group). (E) Ad-Flag-VEGFR2 was mixed with Matrigel prior to injection in the mouse, and the section of the Matrigel was subjected to immunohistochemical evaluation of exogenous VEGFR2 expression using anti-Flag antibody. Scale bars: $20 \mu \mathrm{m}$. (F) Quantitative analysis of in vivo Matrigel plug assay ( $n=3$ per group). (G) Quantitative analysis of in vivo Matrigel plug assay ( $n=3$ per group). Mice received Matrigel supplemented with VEGF-A plus Ad-ME-LA showed increased vessel formation in the absence of FGF signaling. ${ }^{*} P<0.05$ versus respective control or as indicated by brackets.

containing Vegfr2 minimal promoter and the enhancer sequences expressed in BAECs. Introduction of Ad-FGFR1DN resulted in significant reduction in enhancer activity compared with no-Ad or empty Ad (Ad-Null) control that was rescued by expression of ME-LA (Figure 2A). As Erk $1 / 2$ is thought to act by phosphorylating Ets transcription factors, we next sought to identify the cis-acting elements in the Vegfr2 promoter/enhancer affected by FGF-dependent Erk1/2 activation. To this end, we mutated the GG tandem in the core GGAA sequence in 3 Ets binding sites in the Vegfr2 enhancer (Pea3 binding motif, G56T_G57T; Ets1 motif, G303T_G304T; and FOX:ETS composite motif, C378A_C379A).
Enhancer activity assays revealed that only the mutation in the FOX:ETS motif attenuated the activity to a level similar to that achieved by FGF inhibition (Figure 2B). Moreover, the reduced enhancer activity observed in the presence of the mutant FOX: ETS motif was not restored by addition of the constitutively active form of Erk (Figure 2C), which strongly suggests that activation of this FOX:ETS site is directly downstream of Erk $1 / 2$ and critically important for VEGFR2 expression.

To evaluate the capability of Ets family transcription factor binding to the endogenous VEGFR2 enhancer, we performed ChIP assays. We confirmed the binding of Ets 1 and Etv2 to the FOX: 


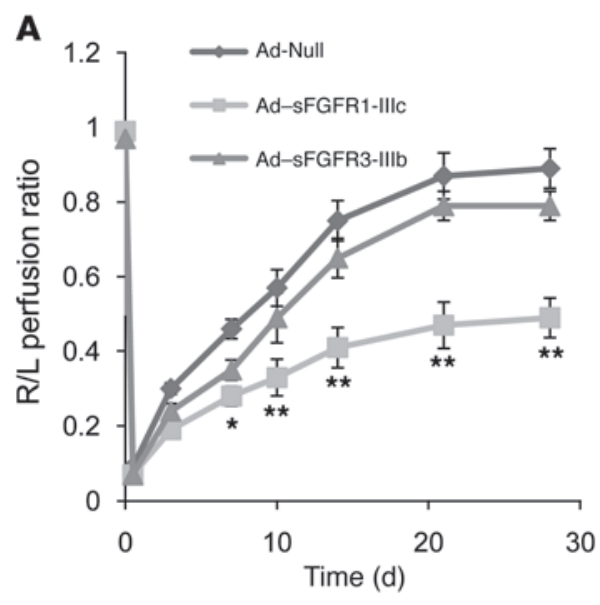

B

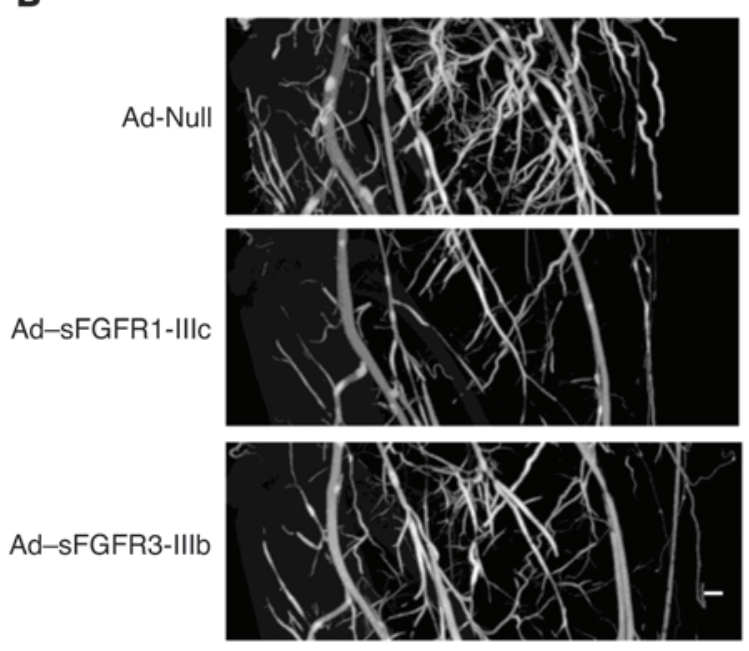

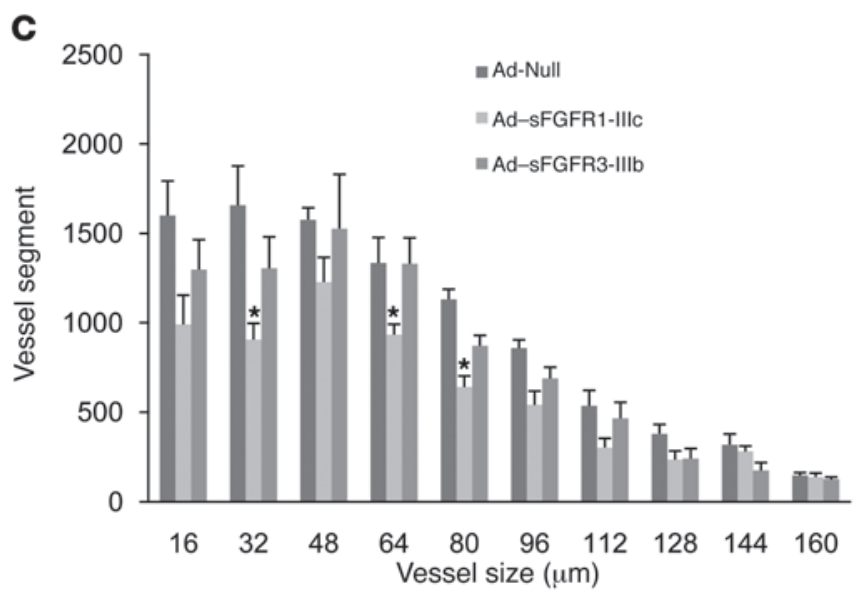

D Nonischemic, Ad-Null Ischemic, Ad-Null Ischemic, Ad-sFGFR1-IIlc
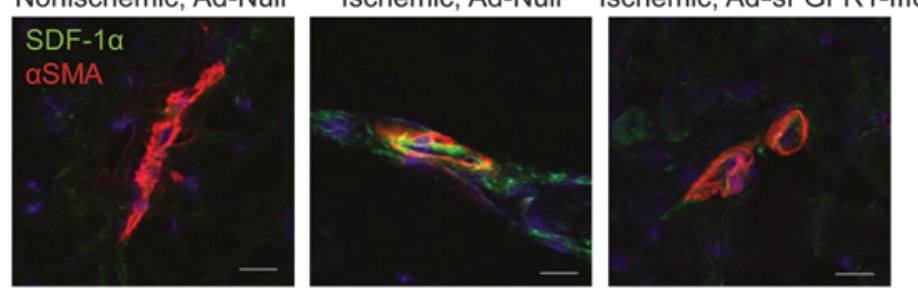

E

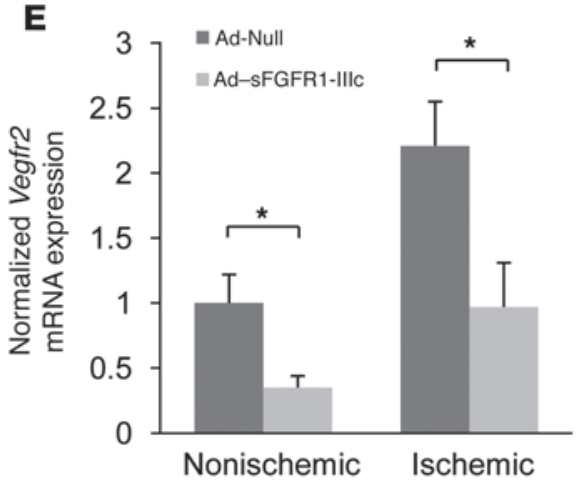

Figure 4

Postischemic neovascularization is impaired in Ad-sFGFR1-IIlc-transduced mice. (A) Laser Doppler analysis of hindlimb perfusion. Ad-Null, Ad-sFGFR1-IIlc, or Ad-sFGFR3-IIIb $\left(5 \times 10^{10}\right.$ viral particle in all cases) was injected 7 days prior to the induction of hindlimb ischemia $(n=6$ per group). Changes in perfusion are shown as a ratio of right to left hindlimb (R/L). Data (mean $\pm \mathrm{SEM}$ ) were analyzed by Student's $t$ test. (B) MicroCT reconstruction at $16-\mu \mathrm{m}$ resolution of the calf portions of a mouse hindlimb 21 days after femoral artery ligation. Scale bar: $273 \mu \mathrm{m} \times 52 \mu \mathrm{m}$. (C) Quantitative analysis of micro-CT images in the calf, presented as total number of vascular structures in $250 \mathrm{z}$ axis slices ( $n=4$ per group). Data are mean \pm SEM. (D) SDF-1 $\alpha$ expression in the small arteries of the ischemic region. Hindlimb ischemia was induced in control and Ad-sFGFR1-IIlc-transduced mice; 48 hours later, gastrocnemius muscle of the ischemic and nonischemic leg was harvested and subjected to immunostaining for SDF-1 $\alpha$ and $\alpha$-SMA. Scale bars: $20 \mu \mathrm{m}$. (E) Quantitative real-time PCR analysis of Vegfr2 expression using total RNA isolated from muscle tissues of C57BL/6 mice injected with Ad-Null or Ad-sFGFR1-IIlc 7 days after ischemia induction $(n=3$ per group). $P<0.05$, ${ }^{* *} P<0.01$ versus Ad-Null.

ETS site of the VEGFR2 enhancer sequence in BAECs (Figure 2D) and MS1 cells (Supplemental Figure 4A). Ets1 Thr38 is an Erk1/2 phosphorylation site thought to be involved in Ets activation (16). Phosphorylation of this site was decreased in BAECs lacking FGF signaling (Supplemental Figure 4B). Treatment with U0126 also decreased Thr38 phosphorylation (Supplemental Figure 4C), demonstrating a link to Erk1/2 signaling. Thus, Ets transcription factors are capable of controlling VEGFR2 transcription via the FOX:ETS motif of the VEGFR2 enhancer and that the FGF-Erk1/2 pathway is required for this regulation.
The possible involvement of FOX:ETS in FGF-dependent regulation of VEGFR2 expression suggests that other endothelial genes whose expression is controlled via the same locus may also be affected. To test this, we examined expression of Tie2, Notch4, and $C d h 5$ (encoding VE-cadherin) in primary ECs transduced with Ad-FGFR1DN. There was a significant decline in both Tie2 and Notch4 expression, whereas $C d h 5$ expression was not changed (Figure 2, E-H). The latter finding is in agreement with our previous study showing that in the absence of FGF signaling, VE-cadherin distribution is severely perturbed, but its expression levels are unchanged (7). 
A
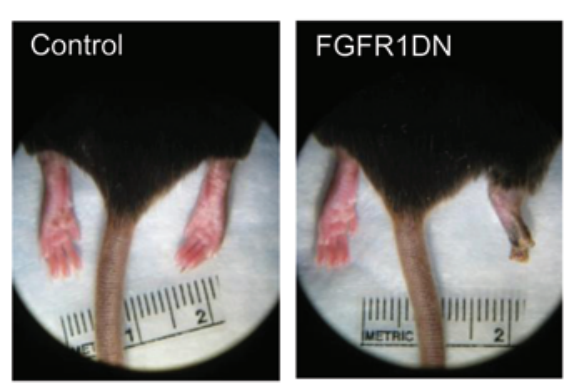

B

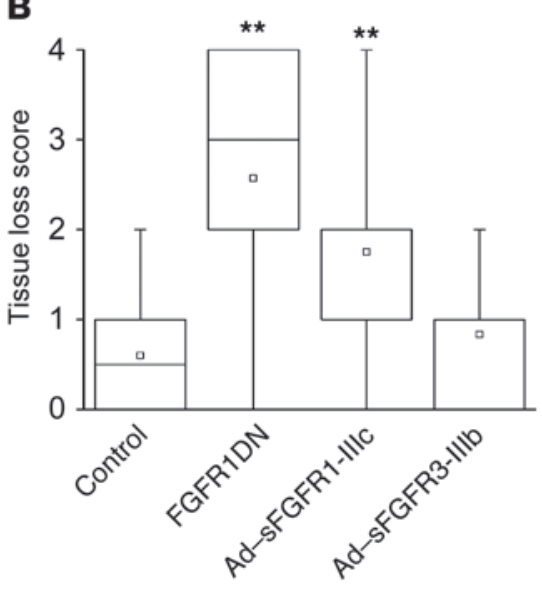

C

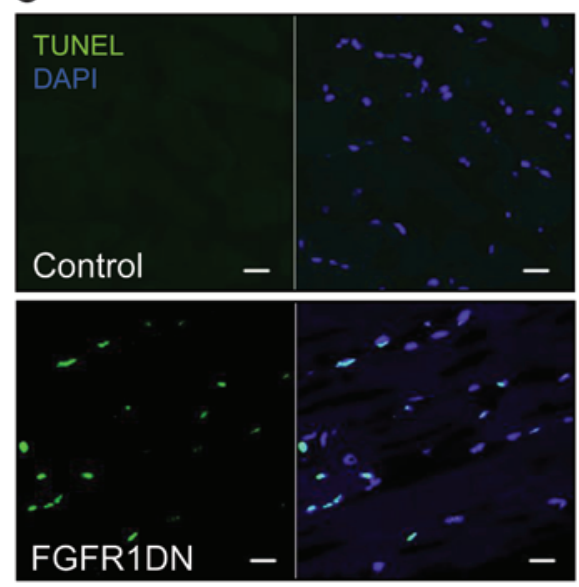

F

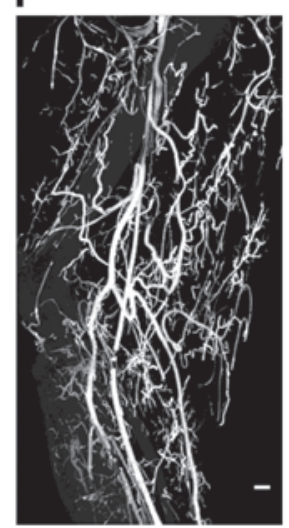

Control

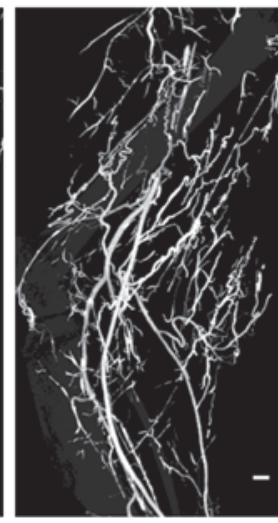

FGFR1DN

G

E

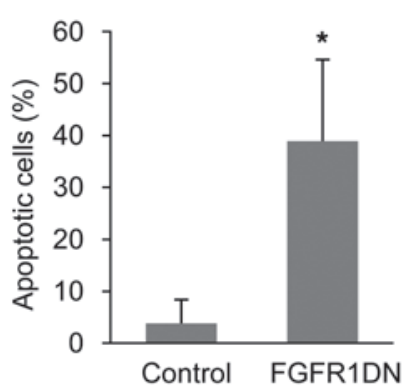

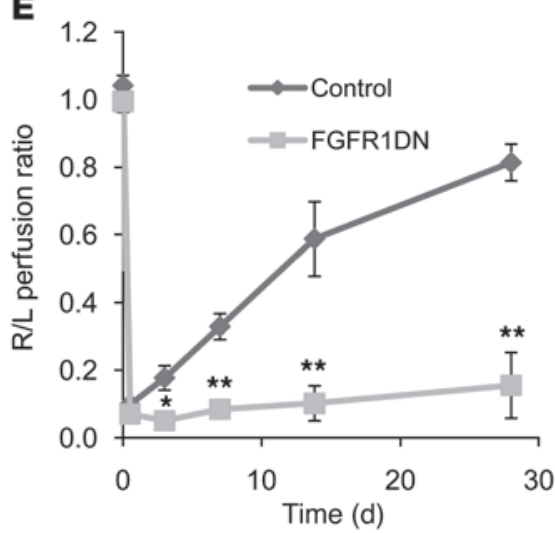

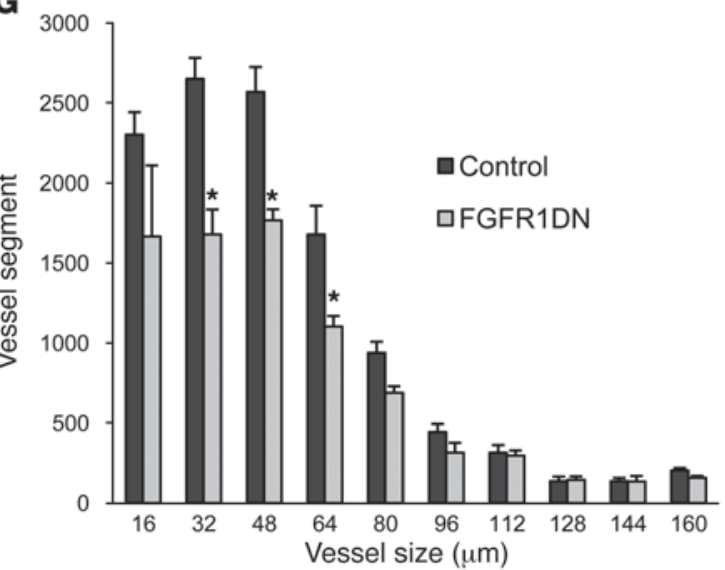

Figure 5

Lack of endothelial FGF signaling impairs postischemic tissue recovery and arteriogenesis. (A) Tissue loss in FGFR1DN mice. After induction of FGFR1DN expression, hindlimb ischemia was produced by ligation of the right femoral artery in control and FGFR1DN mice. Photographs were taken 7 days after induction of ischemia. (B) Tissue loss score (0, healthy; 1 , black nails; 2, black toes; 3, toe loss; 4 , foot loss) at day 7. Data were analyzed by Wilcoxon rank-sum test ( $n=20$ [control]; 14 [FGFR1DN]; 12 [Ad-sFGFR1-IIIc and Ad-sFGFR3-IIIb]). Boxes denote interquartile range; lines within boxes denote median; symbols within boxes denote mean; whiskers denote 5th and 95th percentile; bars denote minimum and maximum. (C) Apoptosis in the ischemic muscle of FGFR1DN mice. 3 days after induction of ischemia, gastrocnemius muscle was taken and stained for TUNEL. Apoptotic nuclei (green) were widely distributed throughout muscle cells of FGFR1DN mice. Scale bars: $20 \mu \mathrm{m}$. (D) Percent TUNEL ${ }^{+}$apoptotic cells relative to DAPI+ cells $(n=3$ per group). (E) Laser Doppler analysis of perfusion in mice after hindlimb ischemia. Changes in perfusion are shown as a ratio of right to left hindlimb ( $n=5$ per group). Data are mean \pm SEM. (F) Micro-CT reconstruction at $16-\mu \mathrm{m}$ resolution of the calf and thigh portions of mouse hindlimbs 21 days after femoral artery ligation. Scale bar: $332 \mu \mathrm{m} \times 96 \mu \mathrm{m}$. (G) Quantitative analysis of micro-CT images in the calf, presented as total number of vascular structures in $250 z$ axis slices ( $n=3$ per group). Data are mean \pm SEM. ${ }^{\star} P<0.05,{ }^{\star \star} P<0.01$ versus respective control. 
A

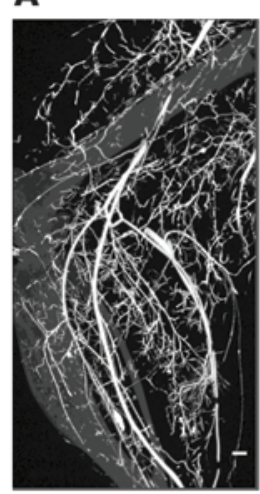

Control

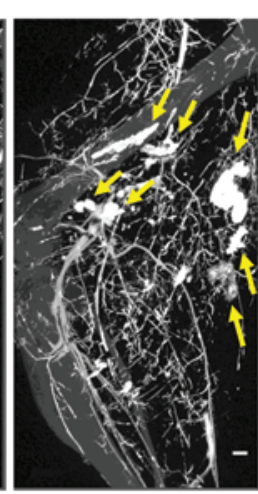

FGFR1DN
B

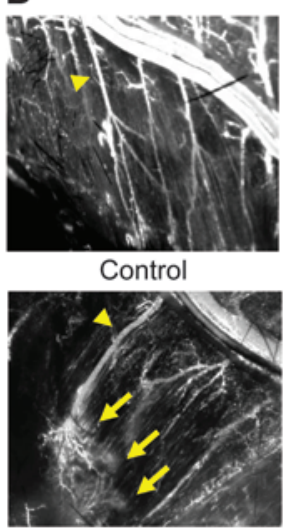

FGFR1DN c

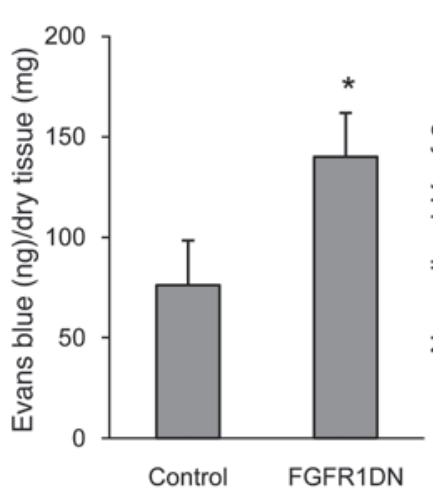

D

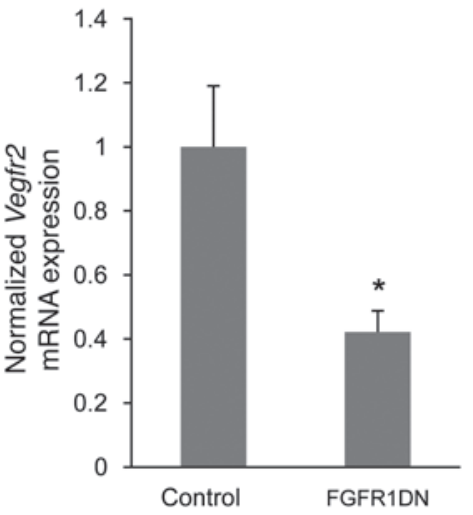

E

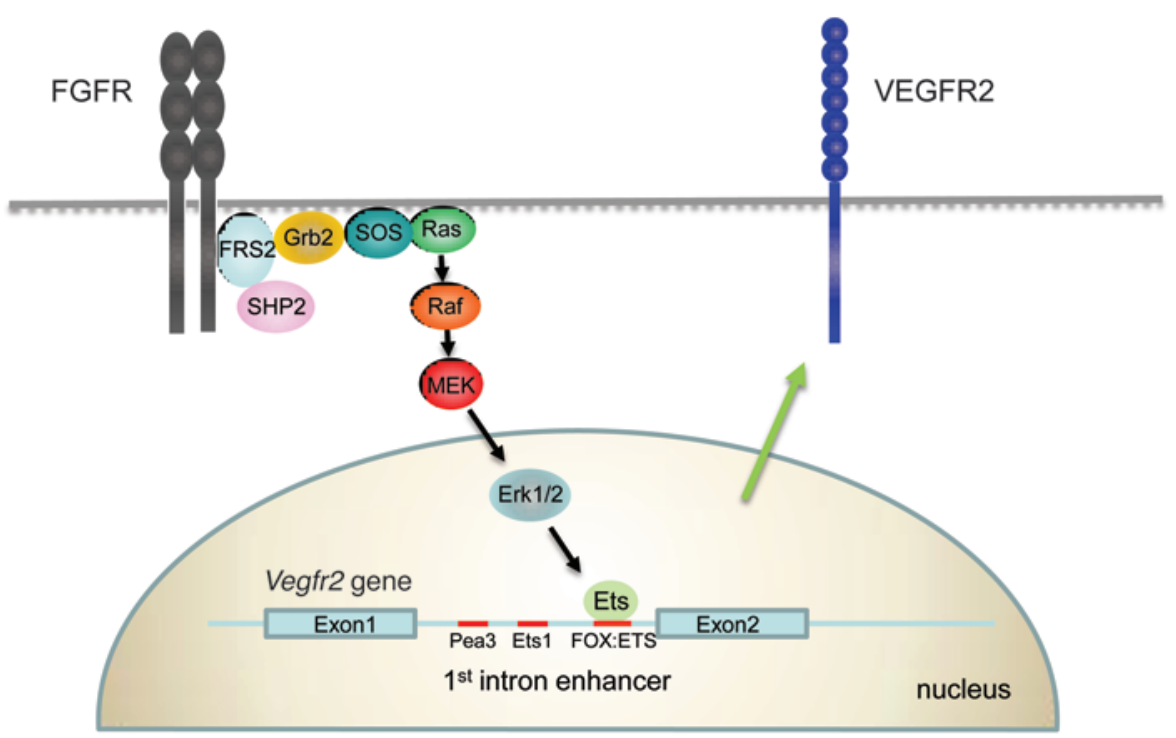

Figure 6

Lack of endothelial FGF signaling impairs vascular integrity. (A) Micro-CT images of arterial vasculature 3 days after femoral artery ligation. Arrows indicate leakage of the contrast agent. Scale bar: $332 \mu \mathrm{m} \times 96 \mu \mathrm{m}$. (B) Vascular leakage in the ischemic thigh of FGFR1DN mice. 3 days after induction of hindlimb ischemia, FITC-dextran $(2 \mathrm{mDa})$ was injected into the carotid artery and visualized with fluorescent stereo-microscope. Arrows indicate sites of dextran extravasation; arrowheads indicate the primary branch of the femoral artery. (C) Quantitative analysis of vascular permeability. Evans blue dye was injected i.v. 3 days after hindlimb ischemia. Mice were then perfused with saline, and the lower part of adductor muscle was taken for quantification ( $n=3$ per group). (D) Quantitative real-time PCR analysis of Vegf2 expression using total RNA isolated from ischemic muscle of control or FGFR1DN mice harvested 7 days after hindlimb ischemia ( $n=3$ per group). ${ }^{*} P<0.05$ versus control. (E) FGF-VEGFR2 interaction. Erk1/2 activated by FGF signaling translocates to the nucleus and promotes Ets binding to the FOX:ETS composite site in the first intron enhancer of Vegfr2. This results in increased VEGFR2 transcription and expression. In the absence of basal FGF signaling, VEGFR2 expression is downregulated, and thus angiogenic activity is decreased.

To further explore the role of FGF-driven Erk1/2 activation in these settings, we analyzed expression of the same genes after transduction with Ad-ME-LA. In agreement with FGFR1DN suppression data, Tie2 and Notch4 expression were induced by ERK, whereas $C d h 5$ expression remained unchanged (Figure 2, I-L). A global analysis of angiogenic gene expression using a small-scale angiogenesis gene array after Ad-FGFR1DN transduction identified a number of genes affected by FGF signaling inhibition, including secreted angiogenic factors, matrix-degrading enzymes, and integrins (Supplemental Figure 5, A-C).

FGF-driven VEGFR2 expression is required for angiogenesis. To determine whether the transcriptional regulation of Vegfr 2 by FGF-Erk $1 / 2$ signal- ing plays a role in angiogenic processes in vivo, we examined VEGFR2 expression in mice with defective FGF signaling by using sFGFR1-IIIc, which we previously demonstrated to virtually shut down FGF signaling (7). At 7 days after intravenous injection of Ad-sFGFR1-IIIc, Vegfr 2 mRNA levels in adductor muscle were significantly decreased compared with those in mice injected with Ad-Null (Figure 3A), which suggests that ongoing FGF signaling is necessary for basal expression of VEGFR2 in vivo. This effect was specific to VEGFR2, since Vegfr $1 \mathrm{mRNA}$ levels were not changed (Figure 3B).

To assess the physiologic significance of this reduction in VEGFR2 expression, Matrigel plugs containing either FGF2 or VEGF-A were implanted subcutaneously in mice injected with 
either Ad-sFGFR1-IIIc or Ad-Null 7 days prior. Blockade of FGF signaling not only attenuated the angiogenic response induced by FGF2, but also inhibited the angiogenic response induced by VEGF-A (Figure 3, C and D), as would be expected given reduced VEGFR2 expression in these settings. To further confirm this, a rescue experiment was performed using Ad-VEGFR2. VEGFR2 expression in the Matrigel plug using a constitutive, non-FGFdependent promoter restored the VEGF-induced angiogenic response in the absence of FGF signaling (Figure 3, E and F). Moreover, the VEGF-A-induced angiogenic response in these settings was also rescued by expression of the ME-LA construct, but not the control ME construct (Figure 3G), further demonstrating that Erk1/2 activation plays an important role in FGF-induced regulation of VEGFR2 expression.

FGF signaling is required for adult arteriogenesis. We next tested the role of FGF-VEGF signaling interplay in arteriogenesis. Hindlimb ischemia studies were carried out in mice expressing either sFGFR1-IIIc (which binds FGF1, FGF2, FGF4, FGF5, FGF6, and FGF8) or sFGFR3-IIIb (FGF1, FGF9, and FGF20) traps (17). Laser Doppler perfusion analyses demonstrated that blood flow recovery started 3 days after ligation in control animals and was essentially complete by day 28 , in agreement with previous publications (18). Flow recovery was significantly impeded in mice with sFGFR1-IIIc, but not sFGFR3-IIIb, FGF traps (Figure 4A). Micro-CT imaging demonstrated reduced arteriogenesis in Ad-sFGFR1-IIIc-injected mice that was limited to smaller arteries (Figure 4, B and C). VEGFmediated SDF-1 expression in the perivascular region plays an important role in promotion of arteriogenesis in this setting (19). Indeed, SDF- $1 \alpha$ was abundantly expressed in perivascular spaces in ischemic tissue in control mice, but its expression was markedly impaired in mice suppressed with FGF signaling (Figure 4D). To examine whether these effects are associated with reduced VEGFR2 expression, we determined Vegfr 2 mRNA expression in Ad-sFGFR1-IIIc-injected and control mice. In agreement with in vitro data, there was a marked decrease in Vegfr 2 mRNA expression not only in normal muscle, but also in ischemic muscle (Figure 4E). Thus, shutdown of FGF signaling in the vasculature caused a decreased arteriogenic response accompanied by downregulation of VEGFR2 expression and reduced VEGF signaling.

Since inhibition of FGF signaling by sFGFR traps may not be limited to ECs, we developed a genetic model of endothelialspecific shutdown of FGF signaling using tetracycline-regulated, endothelial-specific expression of a FGFR1DN construct (referred to herein as TRE-FGFR1DN). To test the activation of FGFR1DN expression by doxycycline withdrawal, TRE-FGFR1DN was expressed in tTA-expressing MEF-3T3 cells. High promoter activity, as measured by $\beta$-galactosidase activity, was seen in the absence of doxycycline and was almost completely suppressed in its presence (Supplemental Figure 6A). Expression of FGFR1DN in the absence of doxycycline was confirmed by Western blotting (Supplemental Figure 6A, inset).

The litter size and body weight of Tie2-tTA/TRE-FGFR1DN double-transgenic mice (FGFR1DN mice) in the "off" mode were similar to those of controls, which indicates that suppression of FGFR1DN expression was successfully achieved during fetal development and in the perinatal growth period. Because expression of the particular type of Tie 2 promoter used in this study is very low in normal adult tissue and significantly stimulated in ischemic tissue (20), we used a hindlimb ischemia model. At 3 days after ischemia induction, there was marked upregulation of endothelial-specific FGFR1DN expres- sion in the affected leg, while it remained undetectable in unaffected muscles (Supplemental Figure 6B). FGFR1DN expression became apparent 24 hours after induction of ischemia, with peak expression at 48 hours (Supplemental Figure 6C).

Induction of hindlimb ischemia after activation of endothelial FGFR1DN expression led to a dramatic phenotype. As early as 3 days after femoral artery ligation, FGFR1DN mice, but not control mice, began exhibiting toe loss; by day 7 , frequent foot loss and severe muscle atrophy were apparent (Figure 5, A and B). Moderate tissue loss was observed in Ad-sFGFR1-IIIc-injected, but not Ad-sFGFR3-IIIb-injected, mice (Figure 5B), and TUNEL staining demonstrated widespread apoptosis of skeletal muscle in the ischemic limb of FGFR1DN mice (Figure 5, C and D). Blood flow analysis using laser Doppler perfusion imaging showed virtually no recovery in FGFR1DN mice, whereas control mice recovered normally (Figure 5E). Micro-CT analysis of arterial vasculature 21 days after induction of ischemia demonstrated a significant reduction in the number of arterial structures $64 \mu \mathrm{m}$ in diameter or smaller, while the number of larger arteries was not affected (Figure 5, F and G). These results suggest that inhibition of the endothelial FGF signaling induced by FGFR1DN expression results in profound disruption of the arteriogenic response.

Early observations in FGFR1DN-expressing mice suggested the presence of edema and hemorrhage in the ischemic limb. Micro-CT imaging at day 3 demonstrated extravasations of contrast (Figure 6A), confirming impaired vessel integrity. To further evaluate this, we obtained FITC-dextran angiograms in the lower adductor group muscle in the ischemic region of FGFR1DN mice and controls. Inhibition of FGF signaling was associated with dextran extravasations (Figure 6B), suggestive of disruption of vessel integrity. In line with the observed loss of arterial integrity, there was a substantial increase in skeletal muscle Evans blue dye retention in FGFR1DN mice compared with control mice (Figure 6C). As in other models, suppression of FGF signaling in these mice was associated with a marked reduction in Vegfr $2 \mathrm{mRNA}$ expression in the ischemic tissue (Figure 6D). Further confirmation of reduced VEGF signaling was shown by profound blunting of a normal increase in tissue cGMP levels (Supplemental Figure 5D).

Inflammatory cells can mediate neovascularization by secreting angiogenic factors in response to angiogenic stimuli. Both FGF and VEGF are capable of recruiting monocytes to the site of new vessel formation, thus augmenting angiogenic response. To test the possibility that FGF inhibition suppresses inflammatory responses, thereby resulting in reduction of VEGFR2 expression and new vessel formation, we evaluated the contribution of inflammatory components to the vascular phenotype observed in our mouse models. In the Matrigel plug, infiltration of CD45 cells was reduced in Ad-sFGFR1-IIIc-injected mice (Supplemental Figure 7, A and B). In contrast, the presence of inflammatory cells in the adductor region of the ischemic leg was comparable in both control and FGFR1DN mice (Supplemental Figure 7, C and D).

\section{Discussion}

Our present data indicate that (a) FGF-induced Erk1/2 activation is required for Ets activation, (b) subsequent Ets binding to the FOX: ETS motif in the first intron enhancer of the Vegfr 2 gene plays an important role in VEGFR2 expression, and (c) this FGF regulation of VEGFR2 expression plays a critical role in adult neovascularization and vascular integrity (Figure 6E). Early observation pointed to critical interplay and synergism of FGF and VEGF systems in angio- 
genic assays, with a combination of VEGF and FGF2 inducing a far stronger in vitro angiogenic response than either growth factor by itself (21). This was thought to be due to an induction of VEGFR2 expression by FGF2 while VEGF induction of FGFR expression was not seen (22). This has been attributed to the involvement of Erk and PKC signaling, but not yet been demonstrated (23). Our data demonstrate, for the first time to our knowledge, that Erk1/2 activation is critical for FGF-dependent regulation of VEGFR2 expression. Moreover, our results indicate that basal endothelial FGF signaling is critically required for VEGF actions that are important for both vessel formation and maintenance. This is in agreement with a prior observation of reduced VEGFR2 expression in $F G F R 1^{-/-}$ embryoid bodies. Surprisingly, however, vascular development in these embryoid bodies was increased (24), perhaps as a result of excessive VEGFR1 activity. In our present study, the consequence of VEGFR2 reduction was attenuation of angiogenic and arteriogenic responses in adult mice.

In many experimental settings, FGF-driven angiogenesis is blocked by VEGF inhibition, which suggests that FGF controls angiogenesis upstream of VEGF by modulating VEGF function $(1,4,25-29)$. This hierarchical regulation appears to play a role similarly in lymphangiogenesis, as FGF2-induced lymphatic growth is inhibited by the blockade of VEGFR3 signaling (30).

Although we demonstrated FGF-VEGF signaling crosstalk in ECs, identifying specific FGF ligands and their source is a more complex issue, since numerous FGFs, including angiogenic (FGF1, FGF2, FGF4, and FGF5) and endocrine (FGF19, FGF21, and FGF23) factors, circulate in the bloodstream. Many of these FGFs can bind to the same FGF receptor subtypes, leading to a high level of redundancy. Given the critical role of FGF-VEGF crosstalk, such redundancy provides obvious evolutionary advantages. In addition to the interaction with the VEGF system, FGFs can control functions of other growth factors and chemokines, such as PDGF, HGF, and MCP-1 (31-34). Although the precise mechanisms of these interactions remain obscure, it is interesting to speculate that FGFs can modulate multiple neovascularization events.

We found that continuous FGF stimulation was necessary for the maintenance of VEGFR2 levels and that in its absence, Vegfr2 expression rapidly declined, leading to reduced production of NO, impaired angiogenesis and arteriogenesis, and, eventually, loss of vascular integrity. This is accomplished at the molecular level by FGF-dependent Erk1/2 activation in the endothelium that in turn leads to Ets family transcription factor binding to the Ets binding site in the recently described FOX:ETS composite site in the Vegfr 2 enhancer (8).

Endothelial enhancers usually have multiple conserved Ets sites, which occur in clusters. In the case of Vegfr 2 , the $3^{\prime}$ region of the first intron $(+3,437 /+3,947)$ contains an autonomous enhancer that drives endothelium-specific Vegfr2 expression (35). Although the enhancer contains at least 3 sites that can bind Ets family members (Pea3, Ets1, and FOX:ETS sites), only the mutation of the Ets core binding sequence in the FOX:ETS site significantly reduced the enhancer's transcriptional activity, which suggests that this is a key site of action. The FOX:ETS site can bind several Ets family members, including Etv2, Ets1, and, likely, Ets2. Etv2 is particularly interesting in light of recent studies showing its involvement in regulation of endothelial-specific gene expression in embryos $(8,36,37)$. The contribution of Etv2 to VEGFR2 expression and embryonic vascular development - through activation of VEGFR2 promoter, but not VEGFR2 enhancer - has been previously demonstrated (36). Etv2 expression is significantly downregulated in adult mice, suggestive of a difference between embryonic and adult angiogenesis (38). Genetic studies show that although neither Ets1-null nor Ets2-null mice exhibit vascular defects, mutation of both Ets1 and Ets 2 results in embryonic lethality as a result of impaired endothelial survival and angiogenesis (39-41). Therefore, it is reasonable to speculate that VEGFR2 expression is controlled by Ets family members in a dynamic fashion. Given the short half-life of VEGFR2 protein, as shown by us and others (ref. 42 and the present study), this finely tuned transcriptional regulation of VEGFR2 expression enables ECs to quickly adjust to changes in the extracellular environment, especially when vessel growth is required.

The role of FGF signaling in the maintenance of VEGFR2 expression is particularly interesting in this context, as augmented and reduced FGF input to the endothelium will lead to increase and decrease, respectively, in VEGFR2 levels, thereby modulating its sensitivity to VEGF stimulation. Our demonstration of FGFdependent maintenance of VEGFR2 expression is consistent with a prior study demonstrating the loss of vascular integrity in the absence of FGF signaling (7) and with studies implicating VEGF signaling in EC survival (43).

FGF-dependent regulation of expression is not limited to VEGFR2. Since the FOX:ETS motif is involved in regulation of a number of endothelial-specific genes $(8,44)$, other genes may be affected as well. Indeed, we observed a reduction in Tie 2 and Notch 4 expression after suppression of FGF signaling, whereas VE-cadherin expression was not affected. The lack of regulation of VE-cadherin expression by the FOX:ETS motif is not surprising, since it is likely that not all FOX:ETS sequences are regulated in the same manner (their regulation is likely to be context dependent), and the relative importance of this motif may vary in different genes.

Although anti-VEGF therapies can cause vessel disintegration and regression not only in many experimental settings, but also in human clinical trials (45), the vasculature quickly rebounds once VEGF inhibition is withdrawn $(46,47)$. A combination of FGF and VEGF signaling inhibition may have a far more profound effect on the vasculature, as the rebound in vascular growth is not likely to occur in the absence of VEGFR2 expression.

In summary, FGF signaling is critically required for the maintenance of VEGFR2 expression, and its inhibition has profound effects on VEGF-dependent biological processes.

\section{Methods}

Study approval. Mice were maintained in the Animal Research Center at Yale University. All animal experiments were performed under a protocol approved by the IACUC of Yale University.

Cell culture and Ad transduction. BAECs and human umbilical artery ECs (Lonza) as well as MAECs and mouse lung microvascular ECs (isolated as previously described; ref. 48 ) were cultured at $37^{\circ} \mathrm{C}$ in $5 \% \mathrm{CO}_{2}$ in EGM-2 MV medium (Lonza) on plates coated with fibronectin $(10 \mu \mathrm{g} / \mathrm{ml})$. Ad vectors were transduced at MOI 10-100 pfu/cell for Ad-FGFR1DN or 100-500 viral particles/cell for Ad-ME and Ad-ME-LA. The infection medium was replaced 4-6 hours later with normal growth medium. cGMP was measured with a cGMP EIA kit (Cayman Chemical).

VEGFR2 enhancer assay and VEGFR2 enhancer mutants. BAECs were transduced with Ad-Null or Ad-FGFR1DN and then transfected with plasmids the next day using Lipofectamine 2000 (Invitrogen). Both transductions and transfections were carried out in triplicate, and each experiment was repeated at least twice. 48-72 hours later, cells were harvested and assayed 
using Dual-Luciferase Reporter Assay System (Promega). Absorbance ratios of Firefly to Renilla luciferases were normalized to the Ad-Null control. Mouse VEGFR2 enhancer/partial promoter construct in PGL2 vector (gift of C. Patterson, University of North Carolina, Chapel Hill, North Carolina, USA; ref. 49) was used for luciferase assay. In mutagenesis studies, the consensus Ets binding core sequence GGAA/T (sense) and A/TTCC (antisense) were mutated to TTAA/T and A/TTAA, respectively. Mutations were introduced at the Pea3 site (ACCAAAGGAAG, G56T_G57T), Ets1 site (GCAGGAAAAC, G303T_G304T), and FOX:ETS site (TTCTTCCTGTTATG, C378A_C379A) with the QuickChange site-directed mutagenesis kit (Stratagene). The VEGFR2 enhancer sequence was based on Genbank accession no. AF061804 (35).

Immunostaining. Muscles from ischemic lower legs were embedded in OCT compound (Sakura Finetek) and frozen at $-80^{\circ} \mathrm{C}$. Cryostat tissue sections $(5-10 \mu \mathrm{m})$ were stained with anti-CD31, -HA, $-\mathrm{SDF}-1 \alpha$, and $-\alpha$-smooth muscle actin antibodies. IgG was used to show antibody specificity.

Hindlimb ischemia model and laser Doppler perfusion imaging. Mouse hindlimb ischemia was induced as described previously (18). Data are reported as a ratio of ischemic (right) to nonischemic (left) limb blood flow (18).

Angiography and micro-CT imaging. Angiography and micro-CT analysis were carried out as described previously (18). Data were expressed as a vascular segment number, representing the total number of vessels of specified diameter counted in $250 z$ sections for calf in 3D micro-CT images.

Permeability assays. $0.5 \%$ Evans Blue $(200 \mu \mathrm{l})$ was injected into the retroorbital space and allowed to circulate for 30 minutes. The mice were then sacrificed, blood was drained, and the adductor group muscle was excised and dried at $55^{\circ} \mathrm{C}$. Evans Blue in tissues was extracted with formamide for 24 hours at $55^{\circ} \mathrm{C}$, and its fluorescence at $607 \mathrm{~nm}$ was measured by a fluorescent reader (Bio-Tek) (7). To visualize vascular leakiness, 3 days after induction of hindlimb ischemia, $500 \mu \mathrm{l} \mathrm{FITC} \mathrm{plus} 2 \mathrm{mDa}$ dextran (Sigma-Aldrich), $10 \mathrm{mg} / \mathrm{ml}$ in $0.1 \times$ PBS, was injected into in the left carotid artery. Dextran extravasation was monitored with a fluorescent stereomicroscope.

Ad-sFGFR infection and in vivo Matrigel plug assay. C57BL/6 mice were injected with $5 \times 10^{10}$ viral particles of Ad-sFGFR1-IIIc or Ad-Null together with $10 \mu \mathrm{M}$ antennapedia peptide (gift of W. Sessa, Yale University; ref. 50).
After 7 days, mice were injected subcutaneously with $0.5 \mathrm{ml}$ Matrigel containing FGF2 $(200 \mathrm{ng} / \mathrm{ml})$ or VEGF-A $(100 \mathrm{ng} / \mathrm{ml})$ with heparin $(10 \mathrm{U})$. Ad-Flag-mVEGFR2, Ad-ME, or Ad-ME-LA $\left(0.85 \times 10^{11}\right.$ viral particles $)$ was added in the Matrigel as described previously (51). 7 days later, mice were euthanized, and Matrigel plugs were stained with a monoclonal CD31 antibody, DYKDDDDK Tag antibody (Cell Signaling), and CD45 antibody (eBioscience). Randomly acquired frames $(368.5 \mu \mathrm{m} \times 368.5 \mu \mathrm{m})$ were analyzed by counting CD $31^{+}$vessels using NIH ImageJ software.

Mouse angiogenesis superarray. Mouse primary lung ECs were transduced with Ad-Null or Ad-FGFR1DN (MOI 50; $n=3$ per group) for 24 hours. Cells were then harvested, and total RNA was collected using RNeasy Plus Mini Kit (Qiagen) and converted to cDNA using High Capacity cDNA Reverse Transcriptase Kit (Applied Biosystems). PCR array was performed using $\mathrm{RT}^{2}$ Profiler PCR Arrays from SABiosciences. Array results were deposited in GEO (http://www.ncbi.nlm.nih.gov/geo/; accession no. GSE28483).

Statistics. Unless otherwise indicated, data are presented as mean \pm SD. Statistical analysis was performed with 2-tailed Student's $t$ test or Wilcoxon rank-sum test. Differences were considered statistically significant for $P$ values of 0.05 or less.

\section{Acknowledgments}

We thank Karen Moodie (Dartmouth Medical School, Hanover, New Hampshire, USA), In Kap Ko (Wake Forest University, Winston-Salem, North Carolina, USA) for technical assistance, and Peter Oettgen (Beth Israel Deaconess Medical Center, Boston, Massachusetts, USA) for comments and suggestions. This study was supported by NIH grant HL 053793 (to M. Simons).

Received for publication August 13, 2010, and accepted in revised form April 13, 2011.

Address correspondence to: Michael Simons, Section of Cardiovascular Medicine, P.O. Box 208017, 333 Cedar Street, New Haven, Connecticut 06520-8017, USA. Phone: 203.785.7000; Fax: 203.785.5144; E-mail: michael.simons@yale.edu.
1. Presta M, Dell'Era P, Mitola S, Moroni E, Ronca R, Rusnati M. Fibroblast growth factor/fibroblast growth factor receptor system in angiogenesis. Cytokine Growth Factor Rev. 2005;16(2):159-178.

2. Beenken A, Mohammadi M. The FGF family: biology, pathophysiology and therapy. Nat Rev Drug Discov. 2009;8(3):235-253.

3. Turner N, Grose R. Fibroblast growth factor signalling: from development to cancer. Nat Rev Cancer. 2010; 10(2):116-129.

4. Murakami M, Simons M. Fibroblast growth factor regulation of neovascularization. Curr Opin Hematol. 2008;15(3):215-220.

5. Dailey L, Ambrosetti D, Mansukhani A, Basilico C. Mechanisms underlying differential responses to FGF signaling. Cytokine Growth Factor Rev. 2005; 16(2):233-247.

6. Eswarakumar VP, Lax I, Schlessinger J. Cellular signaling by fibroblast growth factor receptors. Cytokine Growth Factor Rev. 2005;16(2):139-149.

7. Murakami M, et al. The FGF system has a key role in regulating vascular integrity. J Clin Invest. 2008; 118(10):3355-3366.

8. De Val S, et al. Combinatorial regulation of endothelial gene expression by ets and forkhead transcription factors. Cell. 2008;135(6):1053-1064.

9. Li Y, Basilico C, Mansukhani A. Cell transformation by fibroblast growth factors can be suppressed by truncated fibroblast growth factor receptors. Mol Cell Biol. 1994;14(11):7660-7669.

10. Ueno H, Gunn M, Dell K, Tseng A Jr, Williams L. A truncated form of fibroblast growth factor receptor 1 inhibits signal transduction by multiple types of fibroblast growth factor receptor. J Biol Chem. 1992;267(3):1470-1476.

11. Robinson MJ, Stippec SA, Goldsmith E, White MA, Cobb MH. A constitutively active and nuclear form of the MAP kinase ERK2 is sufficient for neurite outgrowth and cell transformation. Curr Biol. 1998; 8(21):1141-1150.

12. Ren B, et al. ERK1/2-Akt1 crosstalk regulates arteriogenesis in mice and zebrafish. J Clin Invest. 2010; 120(4):1217-1228.

13. Patterson C, Wu Y, Lee ME, DeVault JD, Runge MS, Haber E. Nuclear protein interactions with the human KDR/flk-1 promoter in vivo. Regulation of $\mathrm{Sp} 1$ binding is associated with cell type-specific expression. J Biol Chem. 1997;272(13):8410-8416.

14. Mammoto A, et al. A mechanosensitive transcriptional mechanism that controls angiogenesis. Nature. 2009;457(7233):1103-1108.

15. Sharrocks AD. The ETS-domain transcription factor family. Nat Rev Mol Cell Biol. 2001;2(11):827-837.

16. Foulds CE, Nelson ML, Blaszczak AG, Graves BJ. Ras/mitogen-activated protein kinase signaling activates Ets- 1 and Ets- 2 by CBP/p300 recruitment. Mol Cell Biol. 2004;24(24):10954-10964.

17. Zhang X, Ibrahimi OA, Olsen SK, Umemori H, Mohammadi M, Ornitz DM. Receptor specificity of the fibroblast growth factor family. The complete mammalian FGF family. J Biol Chem. 2006; 281(23):15694-15700.
18. Chittenden TW, et al. Selective regulation of arterial branching morphogenesis by synectin. Dev Cell. 2006;10(6):783-795.

19. Grunewald M, et al. VEGF-induced adult neovascularization: recruitment, retention, and role of accessory cells. Cell. 2006;124(1):175-189.

20. Wong AL, Haroon ZA, Werner S, Dewhirst MW, Greenberg CS, Peters KG. Tie2 expression and phosphorylation in angiogenic and quiescent adult tissues. Circ Res. 1997;81(4):567-574.

21. Pepper MS, Ferrara N, Orci L, Montesano R. Potent synergism between vascular endothelial growth factor and basic fibroblast growth factor in the induction of angiogenesis in vitro. Biochem Biophys Res Commun. 1992;189(2):824-831.

22. Pepper MS, Mandriota SJ. Regulation of vascular endothelial growth factor receptor-2 (Flk-1) expression in vascular endothelial cells. Exp Cell Res. 1998;241(2):414-425.

23. Hata Y, Rook SL, Aiello LP. Basic fibroblast growth factor induces expression of VEGF receptor KDR through a protein kinase $C$ and p44/p42 mitogen-activated protein kinase-dependent pathway. Diabetes. 1999;48(5):1145-1155.

24. Magnusson P, et al. Deregulation of Flk-1/vascular endothelial growth factor receptor-2 in fibroblast growth factor receptor-1-deficient vascular stem cell development. J Cell Sci. 2004;117(pt 8):1513-1523.

25. Seghezzi G, et al. Fibroblast growth factor-2 (FGF-2) induces vascular endothelial growth factor (VEGF) expression in the endothelial cells of forming cap- 
illaries: an autocrine mechanism contributing to angiogenesis. J Cell Biol. 1998;141(7):1659-1673.

26. Tille JC, et al. Vascular endothelial growth factor (VEGF) receptor-2 antagonists inhibit VEGF- and basic fibroblast growth factor-induced angiogenesis in vivo and in vitro. J Pharmacol Exp Ther. 2001;299(3):1073-1085.

27. Auguste $P$, et al. Inhibition of fibroblast growth factor/fibroblast growth factor receptor activity in glioma cells impedes tumor growth by both angiogenesis-dependent and -independent mechanisms. Cancer Res. 2001;61(4):1717-1726.

28. Masaki I, et al. Angiogenic gene therapy for experimental critical limb ischemia: acceleration of limb loss by overexpression of vascular endothelial growth factor 165 but not of fibroblast growth factor-2. Circ Res. 2002;90(9):966-973.

29. Kanda S, Miyata Y, Kanetake H. Fibroblast growth factor-2-mediated capillary morphogenesis of endothelial cells requires signals via Flt-1/vascular endothelial growth factor receptor-1: possible involvement of c-Akt. J Biol Chem. 2004; 279(6):4007-4016.

30. Kubo H, Cao R, Brakenhielm E, Makinen T, Cao Y, Alitalo K. Blockade of vascular endothelial growth factor receptor-3 signaling inhibits fibroblast growth factor-2-induced lymphangiogenesis in mouse cornea. Proc Natl Acad Sci U S A. 2002;99(13):8868-8873.

31. Nissen LJ, et al. Angiogenic factors FGF2 and PDGF$\mathrm{BB}$ synergistically promote murine tumor neovascularization and metastasis. J Clin Invest. 2007; 117(10):2766-2777.

32. Onimaru $\mathrm{M}$, et al. Fibroblast growth factor-2 gene transfer can stimulate hepatocyte growth factor expression irrespective of hypoxia-mediated downregulation in ischemic limbs. Circ Res. 2002;
91(10):923-930.

33. Fujii T, et al. Nonendothelial mesenchymal cellderived MCP-1 is required for FGF-2-mediated therapeutic neovascularization: critical role of the inflammatory/arteriogenic pathway. Arterioscler Thromb Vasc Biol. 2006;26(11):2483-2489.

34. Wempe F, Lindner V, Augustin HG. Basic fibroblast growth factor (bFGF) regulates the expression of the $\mathrm{CC}$ chemokine monocyte chemoattractant protein1 (MCP-1) in autocrine-activated endothelial cells. Arterioscler Thromb Vasc Biol. 1997;17(11):2471-2478.

35. Kappel A, Ronicke V, Damert A, Flamme I, Risau W, Breier G. Identification of vascular endothelial growth factor (VEGF) receptor-2 (Flk-1) promoter/ enhancer sequences sufficient for angioblast and endothelial cell-specific transcription in transgenic mice. Blood. 1999;93(12):4284-4292.

36. Lee D, et al. ER71 acts downstream of BMP, Notch, and Wnt signaling in blood and vessel progenitor specification. Cell Stem Cell. 2008;2(5):497-507.

37. Ferdous A, et al. Nkx2-5 transactivates the Ets-related protein 71 gene and specifies an endothelial/ endocardial fate in the developing embryo. Proc Natl Acad Sci U S A. 2009;106(3):814-819.

38. Brown TA, McKnight SL. Specificities of proteinprotein and protein-DNA interaction of GABP alpha and two newly defined ets-related proteins. Genes Dev. 1992;6(12B):2502-2512.

39. Barton $\mathrm{K}$, et al. The Ets- 1 transcription factor is required for the development of natural killer cells in mice. Immunity. 1998;9(4):555-563.

40. Yamamoto $H$, et al. Defective trophoblast function in mice with a targeted mutation of Ets2. Genes Dev. 1998;12(9):1315-1326.

41. Wei G, et al. Ets1 and Ets2 are required for endothelial cell survival during embryonic angio- genesis. Blood. 2009;114(5):1123-1130

42. Kou R, SenBanerjee S, Jain MK, Michel T. Differential regulation of vascular endothelial growth factor receptors (VEGFR) revealed by RNA interference: interactions of VEGFR-1 and VEGFR-2 in endothelial cell signaling. Biochemistry. 2005; 44(45):15064-15073.

43. Lee $\mathrm{S}$, et al. Autocrine VEGF signaling is required for vascular homeostasis. Cell. 2007;130(4):691-703.

44. De Val S, Black BL. Transcriptional control of endothelial cell development. Dev Cell. 2009; 16(2):180-195

45. Grothey A, Galanis E. Targeting angiogenesis: progress with anti-VEGF treatment with large molecules. Nat Rev Clin Oncol. 2009;6(9):507-518.

46. Kamba T, et al. VEGF-dependent plasticity of fenestrated capillaries in the normal adult microvasculature. Am J Physiol Heart Circ Physiol. 2006;290(2):H560-H576.

47. Mancuso MR, et al. Rapid vascular regrowth in tumors after reversal of VEGF inhibition. J Clin Invest. 2006;116(10):2610-2621.

48. Lanahan AA, et al. VEGF receptor 2 endocytic trafficking regulates arterial morphogenesis. Dev Cell. 2010;18(5):713-724.

49. Wu Y, Moser M, Bautch VL, Patterson C. HoxB5 is an upstream transcriptional switch for differentiation of the vascular endothelium from precursor cells. Mol Cell Biol. 2003;23(16):5680-5691.

50. Gratton JP, et al. Cell-permeable peptides improve cellular uptake and therapeutic gene delivery of replication-deficient viruses in cells and in vivo. Nat Med. 2003;9(3):357-362.

51. Watanabe K, et al. Vasohibin as an endotheliumderived negative feedback regulator of angiogenesis. J Clin Invest. 2004;114(7):898-907. 